\title{
CD40 ligand expression on the surface of colostral $\mathrm{T}$ cells
}

\author{
A Bertotto, G Castellucci, M Radicioni, M Bartolucci, R Vaccaro
}

\begin{abstract}
The proportion of $T$ lymphocytes, mainly CD4 positive, co-expressing the CD40 ligand (CD40-L) was significantly greater $(\mathbf{P}=0.001)$ in the colostrum of 10 breastfeeding mothers than in either autologous or heterologous blood. This surface glycoprotein is a $T$ cell molecule involved in $B$ cell isotype switching and immunoglobulin production with its natural counterreceptor, CD40, expressed by both adult and infant $B$ lymphocytes. As the $T$ cells of newborn infants fail to express the CD40-L when stimulated in vitro, the in vivo upregulation on milk $T$ lymphocytes may be one of the mechanisms through which the mother transfers immune protection to the suckling infant.

(Arch Dis Child 1996; 74: F135-F136)
\end{abstract}

Keywords: colostrum, T cells, CD40 ligand.

Both helper-inducer $\left(\mathrm{CD}^{+}\right)$and suppressorcytotoxic $\left(\mathrm{CD}^{+}\right) \mathrm{T}$ lymphocytes are present in human breast milk. Most of them display the phenotype and functional characteristics of memory $T$ cells, and are activated, as shown by the fact that they express HLA-DR antigens as well as the high affinity interleukin (IL)-2 receptors. ${ }^{1}$ When appropriately stimulated in vitro, adult peripheral blood $\mathrm{T}$ lymphocytes, mostly within the $\mathrm{CD}^{+}{ }^{+}$subset, express the CD40 ligand (CD40-L), an activation molecule involved in B cell isotype switching and immunoglobulin (Ig) production. ${ }^{2}$ As CD40-L expression by newborn cord blood $T$ cells does not occur after in vitro stimulation, ${ }^{2}$ we carried out experiments to determine whether CD40$\mathrm{L}$ is expressed in vivo by colostral $\mathrm{T}$ cells, a cellular component thought to be involved in the lactation transmission of cell mediated immunity. ${ }^{3}$

\section{Methods}

Paired samples of colostrum and peripheral blood were collected from 10 breast feeding mothers between days 4 and 6 after delivery of normal term babies. Heterologous blood from 10 age matched healthy non-pregnant women was also assayed and used as a control. Milk was clarified by shaking with mineral oil. The oily layer was removed and milk cells were sedimented by centrifugation $(600 \times g$, for 15 minutes) from the aqueous layer. The cells were then washed in cold phosphate-buffered saline (PBS) and the lymphocytes purified by centrifuging through 30\% Percoll (Pharmacia Fine Chemicals, Uppsala, Sweden)/PBS. After further washings the lymphoid cells were resuspended in PBS at a final concentration of $3 \times 10^{5} \mathrm{cells} / \mathrm{ml}$. Peripheral blood mononuclear cells from both puerperal and non-pregnant women were isolated by Ficoll-Hypaque (Lymphoprep, Nyegaard, Oslo, Norway) density gradient centrifugation, washed three times, and adjusted to a final concentration of $1 \times 10^{6} \mathrm{cells} / \mathrm{ml}$. Colostral and blood $\mathrm{T}$ cells were phenotyped with the following monoclonal antibodies, used alone or in various combinations: anti-CD3 (OKT3), anti-CD4 (OKT4), anti-CD8 (OKT8) (Ortho, Raritan, New Jersey) and anti-CD40-L (TRAP1, kindly supplied by Dr L D Notarangelo, Department. of Paediatrics, University of Brescia). Surface phenotyping was carried out by single or two-colour indirect immunofluorescence staining techniques which used isotype specific goat anti-mouse antibody (Southern, Biotechnology Associates., Birmingham, Alabama) conjugated with either fluorescein isothiocyanate or phycoerythrin as developing reagent for each monoclonal antibody. The stained cells were fixed in $1 \%$ paraformaldehyde in PBS and analysed in a flow cytometer equipped with a $15 \mathrm{~mW}$ argon-ion laser tuned to $488 \mathrm{~nm}$ (BectonDickinson FACScan, Mountain View, California). In the analysis of single colour and two colour cytofluorimetric data, an electronic gate was set on the lymphocyte population based on the forward angle $v s$ right angle light scatter histogram. Quadrant markers in fluorescence histograms were set using matched isotype controls. The LYSIS II program (BectonDickinson) was used to optimise gating of lymphocytes, and to provide an objective means of excluding both debris (non-cellular events due to particulate matter) and other cell types. After appropriate settings, 20000 cells/test were analysed.

\section{Results}

The proportions of mature $\mathrm{T}$ cells $\left(\mathrm{CD}^{+}\right)$and those of their immunoregulatory $\mathrm{T}$ cell subsets $\left(\mathrm{CD}^{+}\right.$and $\mathrm{CD}^{+}$) were similar in milk and autologous or heterologous blood samples (data not shown). In contrast, the percentage of $\mathrm{CD}^{+}$colostral $\mathrm{T}$ cells coexpressing the CD40-L (TRAP1 ${ }^{+}$) (mean 38\%, SD 6\%) was significantly higher $(P=0.001$, by one way Kruskal-Wallis analysis of the variance) than that calculated in either autologous $(5 \%, 0.5 \%)$ or heterologous $(4 \%, 0.5 \%)$ blood. When the anti-CD3 monoclonal antibody (OKT3) was substituted by one (OKT4) directed against CD4 membrane antigens, two colour cytofluorimetric analysis showed that $87 \%(8 \%)$ of 
the $\mathrm{OKT}^{+}{ }^{+}$milk $\mathrm{T}$ lymphocytes double stained with the TRAP1 monoclonal reagent, whereas only a few $(3 \%)$ circulating $\mathrm{CD}^{+}$ cells did so. The remainder of the TRAP1 ${ }^{+}$ cells in colostrum were $\mathrm{CD}^{+}(5 \%)$, lymphocytes of the non- $T$ lineage, presumably natural killer cells, or occasionally gated monocytes. ${ }^{4}$

\section{Discussion}

The capacity of adult B cells to generate switch events in vivo throughout activation by CD40 molecules requires two different signals: first, a cognate interaction between $T$ and $B$ cells via the CD40-L (present on activated helper $T$ lymphocytes) and CD40 (constitutively expressed by $\mathrm{B}$ cells); and second, $\mathrm{T}$ cell mediated local production of lymphokines, such as IL-4 and IL-10 ${ }^{2}$. CD40/CD40L lymphocyte interplay and co-stimulatory lymphokine secretion are congenitally deficient in human newborn infants. ${ }^{2}$ As a result, in vivo IgG, IgA, and IgE production by neonatal B cells is very low, reaching adult levels only after several months or years. Ineffective Ig isotype switching, a hallmark of the physiological immune deficiency in the neonate, contributes to the observed increased susceptibility to infections during the first few months of life. ${ }^{2}$

Early milk leucocytes are able to resist digestion with trypsin, a characteristic that may permit them to survive in the gastrointestinal tract. ${ }^{5}$ This finding gives credence to animal experiments in which orally administered milk cells were recovered from the gastric and duodenal contents of neonatal mice. These were shown to cross the gut mucosa and enter the circulation of neonatal lambs and the spleen of newborn baboons. ${ }^{5}$ The ability of these immunocompetent cells to survive in the intestine and to secrete their soluble products would allow them not only to potentiate the newborns' bowel immune response, but also their systemic immunoreactivity. We report here that a substantial number of colostral $\mathrm{T}$ cells expresses CD40-L. Others have reported that these lymphocytes synthetise both IL-4 and $\mathrm{IL}-10^{6}$. When viewed together, these findings sustain the concept that natural protective mechanisms help to endow the immunocompromised baby with extrinsic immunological support and, therefore, underline the usefulness of breast feeding in preventing neonatal infections.

1 Wirt DP, Adkins LT, Palkowetz KH, et al. Activated and memory T lymphocytes in human milk. Cytometry 1992; 13: $282-90$

2 Brugnoni D, Airò P, Graf D, et al. Ineffective expression of CD40 ligand on cord blood $\mathrm{T}$ cells may contribute to poor immunoglobulin production in the newborn. Eur $\mathcal{f}$ Immunol 1994; 24: 1919-24.

3 Bertotto A, Gerli R, Fabietti G, et al. Human breast milk T lymphocytes display the phenotype and functional characteristics of memory T cells. Eur $\mathcal{f}$ Immunol 1990; 20: teristics $1877-80$.

4 Gruss HJ, Dower SK. The TNF ligand superfamily and its relevance for human diseases. Cytokines and Molecular Therapy 1995; 1: 75-105.

5 Jain L, Vidyasagar D, Xanthou M, Ghai V, Shimada S, Blend $M$. In vivo distribution of human milk leucocytes after ingestion by premature baboons. Arch Dis Child 1989; 64: 930-3.

6 Akansén-Saphir U, Lindfors A, Andersson U. Cytokine production in mononuclear cells of human milk studied at the single-cell level. Ped Res 1993; 34: 213-6. 